\title{
MODELS OF KIN SELECTION ON SEED PROVISIONING
}

\author{
DAVID C. QUELLER* \\ School of Biological Sciences, University of Sussex, Falmer, Brighton BN1 9QG, England
}

Received 21.xi.83

\section{SUMMARY}

\begin{abstract}
ESS models support the prediction of Westoby and Rice (1982) and Queller (1983), based on relatedness arguments, that genes affecting the amount of parental resources to be invested in seeds will be selected towards different outcomes, depending on the tissue in which they are expressed. In order of decreasing "preference" for investment in their own seed, the tissues generally rank as: embryo, endosperm, gametophyte, maternal plant. A more detailed model reveals that whether or not selection will favour an endosperm allele for taking more investment depends on frequency and dosage. These effects are analogous to those arising from inbreeding in other kin selection models, and they disappear when a covariance form of the relatedness coefficient is used.
\end{abstract}

\section{INTRODUCTION}

The genetic complexities of seed development, particularly in the angiosperms or flowering plants, have long puzzled botanists. Briefly (see Maheshwari, 1950, for details), a cell of the diploid maternal plant undergoes meiosis, one of the daughter cells proliferates into a multicellular, haploid gametophyte, and at least one of the gametophytic cells functions as an egg and is fertilised by a pollen nucleus to begin the new diploid embryo. There are therefore three generations participating in the developing seed. In the gymnosperms, the gametophyte functions in acquiring nutrients for its embryo from the maternal plant. In the angiosperms, the gametophyte is ephemeral and is supplanted in its nutrient-acquiring role by a unique genetic entity, the endosperm. There are several variations in endosperm composition, but I will focus here on the most common, presumably ancestral (Maheshwari, 1950), type. It is formed by the fusion of two maternal gametophytic nuclei and a pollen nucleus identical to the one fertilising the egg cell (there is usually only one endosperm and one embryo in an angiosperm seed). The endosperm is therefore genetically identical to the embryo except that it carries a double dose of maternal alleles.

Charnov (1979) was the first to provide a concrete suggestion on how kin selection might influence the evolution and function of the endosperm and the idea was developed by Westoby and Rice (1982) and Queller (1983). This kin selection approach rests on a foundation of resource trade-offs. It is assumed that when resources go to one embryo, there is a cost to other embryos on the same maternal plant, either by directly reducing the pool of available resources, or by increasing the parent's risk of mortality and thereby lowering the expected number of future embryos. Following Hamilton (1964), Trivers (1974) showed how such trade-offs, coupled with kin selection, can lead to conflicts between parents and offspring over the distribution of parental investment (PI).

* Present address: 2406 S Prospect Ave, Champaign IL 61820 U.S.A. 
The arguments of Westoby and Rice (1982) and Queller (1983) simply extend Trivers' logic to the gametophyte and the endosperm. Selection on any of the entities potentially able to influence the amount of PI given to a seed (maternal plant, gametophyte, endosperm, embryo) will be determined by the opportunities that each has for getting its genes into the next generation, that is by the likelihood that its genes will be transmitted by provisioning its own embryo, and by the likelihood that they will be transmitted if the resources are allowed to go to other embryos. By a simple extension of Hamilton's (1964) rule, an individual should aid one relative at the expense of another when $b r_{b}>c r_{c}$, where $b$ is the benefit to one, $c$ is the cost to the other, and $r_{b}$ and $r_{c}$ are the individual's coefficients of relatedness to the relatives obtaining the benefit and the cost respectively (West-Eberhard, 1975). Application of this rule to the "individuals" involved in seed development yields the conditions listed in table 1 . When embryos on the same plant are at least sometimes half-sibs rather than full-sibs, as must usually be the case, the four genetic entities can be ranked according to the extent to which they should be selected to favour provisioning for their own seed at the expense of other seeds. This ranking, with the embryo favouring its own seed the most, followed in order by the endosperm, the gametophyte, and the parent, may account for many features of seed development, including the evolution and function of the endosperm (Westoby and Rice, 1982; Queller, 1983).

TABLE 1

Conditions, based on relatedness arguments (Westoby and Rice, 1982; Queller, 1983), under which the various genetic entities in the seed should be selected to aid the embryo of its own seed (with benefit b) at the expense (c) of other embryos on the same plant

\begin{tabular}{lcc}
\hline & \multicolumn{2}{c}{ costs fall on: } \\
& half-sib embryos & full-sib embryos \\
\hline maternal & $b>c$ & $b>c$ \\
gametophyte & $b>c / 2$ & $b>c / 2$ \\
endosperm & $b>c / 3$ & $b>c / 2$ \\
embryo & $b>c / 4$ & $b>c / 2$ \\
\hline
\end{tabular}

However, arguments based on relatedness are not always precisely supported by more detailed models of changes in gene frequency (e.g., Williams and Williams, 1957; Maynard Smith, 1965; Levitt, 1975; Charlesworth, 1978; Macnair and Parker, 1979). Law and Cannings (unpublished ms.) have modelled kin-selected conflicts in seeds and showed that the results may not match those predicted simply on the basis of relatedness. In this paper, I will present models which show that, under the appropriate conditions, relatedness arguments do provide fairly good guides to the outcome of selection, though selection on endosperms does show some peculiar features.

Two models will be developed. The first extends previous models of parent-offspring conflict (Parker and Macnair, 1978; Macnair and Parker, 1978) to include gametophytes and endosperms, and searches for evolutionary stable strategies (Maynard Smith, 1982). The second model is less rigorous in one of its assumptions, but it allows further exploration of some 
of the peculiar features of endosperm selection brought out by the first model.

\section{AN ESS MODEL}

The following model is an extension of the parent-offspring models of Parker and Macnair (1978) and Macnair and Parker (1978), with some minor changes in notation adopted by Parker (unpublished ms.). I will assume a large population of hermaphroditic, outcrossing plants (the Parker and Macnair models are for dioecious organisms, but the formal structure is not altered). Selection is assumed to be weak. Generations are discrete, but seeds are not all produced simultaneously. Seeds germinate in the first available growth period. Each plant has a fixed amount, $M$, of parental investment to distribute among a large number of seeds.

The model solves for the evolutionarily stable amount of parental investment, $m_{*}$, to be solicted or taken by embryos (or by endosperms or gametophytes for their own embryos). Genetic variants are assumed to differ only in the amount of PI (parental investment) they obtain. Any given amount, $m$, of PI results in an embryo of fitness $f(m)$ which is most simply viewed as its probability of survival to reproductive age, though it could be defined to include fecundity effects. Two assumptions are made about the shape of $f(m)$. First, it reaches a point of diminishing returns for increased $m$ (i.e., the second derivative with respect to $m$ is negative). If it did not, the parent would gain by putting all its PI into a single offspring (Smith and Fretwell, 1974) and such a species would be unlikely to persist. I will also assume that $f(m)$ is monotonic increasing in the range of interest. Although it is possible that an offspring could get so much PI that its personal fitness is lowered, the detriment to siblings would halt acquisition before it evolved to this point.

The cost of selfishness is assumed to fall not on contemporary embryos (this case is modelled by Law and Cannings, unpublished Ms.) but on later embryos. Specifically, the maternal parent will be able to mature fewer later seeds than it would otherwise have done.

The evolutionarily stable strategy for the parent is to put $m_{*}=$ $f\left(m_{*}\right) / f^{\prime}\left(m_{*}\right)$ units of PI into each offspring that it raises, and the stable offspring strategy is to take $m_{*}=f\left(m_{*}\right) / 4 f^{\prime}\left(m_{*}\right)$ or $m_{*}=f\left(m_{*}\right) / 2 f^{\prime}\left(m_{*}\right)$, depending on whether the future embryos being displaced by selfish acquisition are half-sibs or full-sibs to the embryos getting the benefit (Parker and Macnair, 1978; Macnair and Parker, 1978). It has not been pointed out that these results match Hamilton's rule. For example, the offspring rule for full-sib displacement can be written as $f^{\prime}\left(m_{*}\right)=f\left(m_{*}\right) / 2 m_{*}$ or $\mathrm{d} f=$ $f\left(m_{*}\right) \mathrm{d} m / 2 m_{*}$ which is equivalent to $b=c / 2$. The benefit, or $d f$, is the marginal increase in fitness of an offspring taking $d m$ extra units of PI. Since the cost is in the form of having fewer future sibs, the rate of loss of sibling fitness is $f\left(m_{*}\right)$ for every $m_{*}$ units of PI taken by earlier sibs. The average cost of a single early sib taking $d m$ more units of PI is therefore $f\left(m_{*}\right) d m / m_{*}$.

\section{(i) Gametophytic ESS}

Let $\boldsymbol{A}_{*}$ represent a gametophytic allele that takes an amount $m_{*}$ of PI for its embryo. If $m_{*}$ is an ESS then the $A_{*}$ allele must transmit more copies 
of itself to the next generation than any rare mutant $A_{m}$ allele that takes an amount of PI, $m_{m}$, differing slightly from $m_{*}$. Since $A_{m}$ is rare, the frequency of $A_{m} A_{m}$ genotypes will be negligible, and we must compare the number of copies of $A_{m}$ left by an $A_{m} A_{*}$ adult with the number of copies transmitted by the typical $A_{*}$ allele, i.e., one in an $A_{*} A_{*}$ adult. Therefore, $A_{*}$ is stable against invasion when:

$$
\frac{\frac{1}{2} M f\left(m_{m}\right)}{\frac{1}{2}\left(m_{m}+m_{*}\right)}<\frac{\frac{1}{2} M f\left(m_{*}\right)}{m_{*}},
$$

for all $m_{m}$ near $m_{*}$. On the L.H.S., the offspring number of an $A_{m} A_{*}$ mother is her total amount of PI, $M$, divided by the average amount faken by her gametophytes, $\frac{1}{2}\left(m_{m}+m_{*}\right)$. Of these offspring, half bear the $\boldsymbol{A}_{m}$ allele, and they have a survival rate of $f\left(m_{m}\right)$. On the R.H.S., $A_{*} A_{*}$ mothers have $M / m_{*}$ offspring, surviving at the rate of $f\left(m_{*}\right)$, half of which bear any given maternal $A_{*}$ allele. Here it is unnecessary to consider fitness through the paternal side because the gene in question is expressed only in maternal gametophytes.

Letting $D$ stand for the difference, L.H.S.-R.H.S., formula 1 is true for all $m_{m} \rightarrow m_{*}$ if $D$ is at a local maximum (of zero) when $m_{m}=m_{*}$, i.e., when:

and

$$
\left.\frac{d D}{d m_{m}}\right|_{m_{m}=m_{*}}=0
$$

$$
\left.\frac{d^{2} D}{d m_{m}^{2}}\right|_{m_{m}=m_{*}}<0 \text {. }
$$

If 2 and 3 hold, any small deviation from $m_{*}$ will result in fewer copies of the mutant allele being transmitted. Formula 2 yields $f^{\prime}\left(m_{*}\right)=f\left(m_{*}\right) / 2 m_{*}$ or $b=c / 2$, in agreement with simple relatedness arguments (table 1 ). Letting $f^{\prime \prime}(m)$ stand for the second derivative of the fitness function, formula 3 is equivalent to:

$$
\frac{M 2 m_{*}^{2} f^{\prime \prime}\left(m_{*}\right)-M\left[2 m_{*} f^{\prime}\left(m_{*}\right)-f\left(m_{*}\right)\right]}{m_{*}}<0 .
$$

Since $f^{\prime}\left(m_{*}\right)=f\left(m_{*}\right) / 2 m_{*}$ and $f^{\prime \prime}\left(m_{*}\right)<0$, this expression always holds and formula 2 therefore defines a local maximum and an ESS. This result, $b=c / 2$, holds for costs falling on either half-sib or full-sib embryos since formula 1 holds for both (because the gametophyte is equally related to its embryo's half and full siblings).

\section{(ii) Endosperm ESS}

The same method of finding a local maximum of a difference function, $D$, will be employed to search for an endosperm ESS, but the equation is more complex. Paternal reproduction must be incorporated since endosperm alleles transmitted through pollen can be expressed. This also means that the half-sib and full-sib cases must be treated separately.

In addition, since with two allelomorphs there are four possible endosperm genotypes, two dosage parameters must be used. If the homozygous normal endosperm, $A_{*} A_{*} A_{*}$, takes $m_{*}$ units of PI for its embryo, and the 
homozygous mutant, $A_{m} A_{m} A_{m}$, takes $m_{m}$ units, let the number of units taken by $A_{m} A_{*} A_{*}$ and $A_{m} A_{m} A_{*}$ endosperms be $m_{*}+h_{1}\left(m_{m}-m_{*}\right)$ and $m_{*}+$ $h_{2}\left(m_{m}-m_{*}\right)$ respectively. Thus, $h_{1}$ and $h_{2}$ represent the fractional expression of endosperms with one and two mutant alleles.

Unless $h_{1}$ and $h_{2}$ are very small (see the recessive case treated separately below), the spread of a mutant allele when rare will be determined by the success of $A_{m}$ alleles in $A_{m} A_{*} \times A_{*} A_{*}$ matings compared with the success of $A_{*}$ alleles in $A_{*} A_{*} \times A_{*} A_{*}$ matings. Assuming first that pollen dispersal is random so that the embryos on a plant are typically half sibs, the difference between the average numbers of copjes left by $A_{m}$ and $A_{*}$ alleles when $A_{m}$ is rare is

$$
D=\frac{\frac{1}{2} M f\left[m_{*}+h_{1}\left(m_{m}-m_{*}\right)\right]}{m_{*}}+\frac{\frac{1}{2} M f\left[m_{*}+h_{2}\left(m_{m}-m_{*}\right)\right]}{\frac{1}{2}\left[2 m_{*}+h_{2}\left(m_{m}-m_{*}\right)\right]}-\frac{\frac{1}{2} \cdot 2 M f\left(m_{*}\right)}{m_{*}} .
$$

The last term, as in the gametophytic case, is the $A_{*}$ term, but doubled since male reproduction is being included. The first two terms represent the number of copies left by a rare $\boldsymbol{A}_{m}$ allele through male and female reproduction, respectively. Half of an $A_{m} A_{*}$ 's progeny through pollen will have an endosperm/embryo genotype of $A_{m} A_{*} A_{*} / A_{m} A_{*}$ with offspring fitness of $f\left(m_{*}+h_{1}\left(m_{m}-m_{*}\right)\right)$. The number of seeds sired will be no lower than normal $\left(M / m_{*}\right)$ because the costs of "selfishness" fall on half siblings which are $A_{*} A_{*}$ when $A_{m}$ is rare. The number of seeds produced through female reproduction of an $A_{m} A_{*}$ is changed because the average PI taken by its $A_{m} A_{m} A_{*}$ and $A_{*} A_{*} A_{*}$ endosperms is $\frac{1}{2}\left(2 m_{*}+h_{2}\left(m_{m}-m_{*}\right)\right)$.

Substituting equation 4 into equation 2 yields

$$
f^{\prime}\left(m_{*}\right)=\frac{h_{2}}{2\left(h_{1}+h_{2}\right)} \cdot \frac{f\left(m_{*}\right)}{m_{*}}
$$

or $b=c h_{2} /\left(2 h_{1}+2 h_{2}\right)$. Substituting $D$ into formula (3) gives $M f^{\prime \prime}\left(m_{*}\right) \times$ $\left(h_{1}^{2}+h_{2}^{2}\right) / m_{*}<0$, which is true, indicating that (5) gives a local maximum. The prediction of simple relatedness arguments, that an allele cannot be selected unless $b>c / 3$, is supported for mutant alleles with additive dosage effects $\left(h_{1}=\frac{1}{2}, h_{2}=\frac{2}{3}\right)$. However, the $m_{*}$ defined by (5) is not really an ESS because it varies with the dosage parameters of the mutant. For example, it is stable against invasion by any dominant mutant $\left(h_{1}=h_{2}=1\right)$ only when $b=c / 4$. However the qualitative prediction that endosperm alleles will be selected to be more aggressive in obtaining PI than gametophytes but less aggressive than offspring appears to be supported by the model because, except in the unlikely event that $h_{1}>h_{2}$, the conditions defined by (5) range between $b=c / 2$ and $b=c / 4$, which correspond to the gametophytic and offspring ESS's.

While it seems implausible that the sibs displaced by "selfish" endosperms would always be full sibs to the ones aided, it is useful to consider it as a limiting case. Here the difference function is

$$
D=\frac{\frac{1}{2} M f\left[m_{*}+h_{1}\left(m_{m}-m_{*}\right)\right]}{\frac{1}{2}\left[2 m_{*}+h_{1}\left(m_{m}-m_{*}\right)\right]}+\frac{\frac{1}{2} M f\left[m_{*}+h_{2}\left(m_{m}-m_{*}\right)\right]}{\frac{1}{2}\left[2 m_{*}+h_{2}\left(m_{m}-m_{*}\right)\right]}-\frac{\frac{1}{2} \cdot 2 f\left(m_{*}\right)}{m_{*}}
$$

which differs from (4) only in the denominator of the first term; paternallyderived $\boldsymbol{A}_{\boldsymbol{m}}$ alleles now occur together in the progeny of a single maternal 
plant $\left(A_{*} A_{*}\right)$. Substitution of this into (2) yields

$$
f^{\prime}\left(m_{*}\right)=\frac{f\left(m_{*}\right)}{2 m_{*}}
$$

or $b=c / 2$, in agreement with table 1 . The second derivative is the same as in the half-sib case, again indicating a maximum.

\section{(iii) Endosperm recessive mutants}

Though Parker and Macnair (1978) used a somewhat different method for recessive alleles, I will continue with the approach used above. If an endosperm mutant is recessive such that three copies are required for its effect to be expressed $\left(h_{1}=h_{2}=0\right)$, then the important mutant mating to consider is $A_{m} A_{*} \times A_{m} A_{*}$ (Parker and Macnair, 1978). Since each such mating requires one heterozygote acting as a male and one acting as a female, it is not necessary to consider male and female reproduction separately.

In contrast to the other cases, there will be both $A_{m} A_{*}$ and $A_{m} A_{m}$ offspring involved and it is necessary to consider the effect of $A_{*} A_{*} A_{*}$ endosperms on the former even though their own endosperms do not express the mutant trait. Assume first that pollen dispersal is random so that all embryos on a plant are half siblings. In addition, since $A_{m}$ is rare, assume that each heterozygote is pollinated by no more than one $A_{m}$ pollen grain (from another heterozygote). Letting $n$ stand temporarily for the number of seeds matured by a heterozygous plant pollinated by one $A_{m}$ pollen grain and $n-1 A_{*}$ grains, the difference function is

$$
D=f\left(m_{m}\right)+\frac{1}{2} \cdot \frac{1}{2}(n-1) f\left(m_{*}\right)-\frac{M f\left(m_{*}\right)}{m_{*}} .
$$

The first term is for the single $A_{m} A_{m}$ offspring which transmits one copy of the mutant allele for every one of its parents' copies. The second term is for the $\frac{1}{2}(n-1)$ heterozygous offspring who carry one mutant allele for every two parental mutant alleles. The paternal $A_{m}$ must be included even though the heterozygous offspring have different fathers because, without its presence, there would be no effect at all on heterozygous offspring. The effect on heterozygous offspring is a change in their number. Recall that the number of seeds matured is the total amount of PI divided by the average amount taken, so $n$ can be defined recursively as

$$
m=\frac{M}{\frac{1}{n}\left(m_{m}\right)+\frac{n-1}{n}\left(m_{*}\right)}
$$

Solving for $n-1$ yields $\left(M-m_{m}\right) / m_{*}$, which can be substituted into (7) above. Substituting that in turn into (2) gives

$$
f^{\prime}\left(m_{*}\right)=\frac{f\left(m_{*}\right)}{4 m_{*}}
$$

or $b=c / 4$. Substitution of (7) into (3) gives $f^{\prime \prime}\left(m_{*}\right)$, which is negative, indicating an ESS. A recessive endosperm mutant, like a dominant one, is selected as if it were an offspring allele. 
Finally, consider a recessive endosperm allele for the limiting case in which all the embryos on a plant are fathered by the same paternal plant. The progeny of a $A_{m} A_{*} \times A_{m} A_{*}$ mating would consist of $\frac{1}{4} A_{m} A_{m} A_{m} / A_{m} A_{m}$, $\frac{1}{4} A_{m} A_{m} A_{*} / A_{m} A_{*}, \frac{1}{4} A_{m} A_{*} A_{*} / A_{m} A_{*}$ and $\frac{1}{4} A_{*} A_{*} A_{*} / A_{*} A_{*}$, with only the first type expressing the mutant trait. If $n$ is the number of progeny from such a mating, the difference function is

$$
D=\frac{1}{4} n f\left(m_{m}\right)+\frac{1}{2} \cdot \frac{1}{2} n f\left(m_{*}\right)-\frac{M f\left(m_{*}\right)}{m_{*}} .
$$

Here $n=M /\left(m_{m} / 4+3 m_{*} / 4\right)$. Substituting $n$ into equation (8), and $D$ from equation (8) into equation (2) yields

$$
f^{\prime}\left(m_{*}\right)=\frac{f\left(m_{*}\right)}{2 m_{*}}
$$

or $b=c / 2$. From (8) and (3),

$$
\left.\frac{d^{2} D}{d m_{m}^{2}}\right|_{m_{m}=m_{*}}=\frac{4 m^{2} f^{\prime \prime}\left(m_{*}\right)-2 m_{*} f^{\prime}\left(m_{*}\right)+f\left(m_{*}\right)}{32 m_{*}^{3}}
$$

which, when (9) holds, equals $f^{\prime \prime}\left(m_{*}\right) / 8 m_{*}$, indicating an ESS.

\section{(iv) Offspring ESS}

Parker and Macnair (1978) showed that the offspring strategy defined by $f^{\prime}\left(m_{*}\right)=f\left(m_{*}\right) / 4 m_{*}$ is stable against dominant and recessive mutants when half-sibs bear the costs. However, it has now been shown that the strategy stable against endosperm mutants depends on dosage, even though it does not differ for dominant and recessive mutants. To see if this property is peculiar to the endosperm, the offspring strategy stable against partially dominant mutants is derived below.

If normal $A_{*} A_{*}$ offspring take $m_{*}$ units of PI, and homozygous mutants take $m_{m}$ units, let the number of units taken by heterozygotes be defined as $m_{*}+h\left(m_{m}-n_{*}\right)$. Unless $h$ is very small, the fate of a rare $A_{m}$ allele depends on $A_{m} A_{*} \times A_{*} A_{*}$ matings. Following the same logic as before, if only half sibs suffer the costs of "selfish" behaviour the difference equation is

$$
D=\frac{\frac{1}{2} M f\left[m_{*}+h\left(m_{m}-m_{*}\right)\right]}{m_{*}}+\frac{\frac{1}{2} M f\left[m_{*}+h\left(m_{m}-m_{*}\right)\right]}{\frac{1}{2}\left[2 m_{*}+h\left(m_{m}-m_{*}\right)\right]}
$$

Substituting this into (2) and (3) yields $f^{\prime}\left(m_{*}\right)=f\left(m_{*}\right) / 4 m_{*}$ and $M h^{2} f^{\prime \prime}\left(m_{*}\right)<$ 0 . Since the second condition is true, the first defines an ESS, which does not depend on dosage.

\section{A MORE GENERAL ENDOSPERM MODEL}

The endosperm behaviour predicted by the preceding models is peculiar in being dependent on dosage, in not stabilising at a particular ESS, and in not precisely matching the expectation based on relatedness. I will therefore present another model of endosperm selection which, though less rigorous in some of its assumptions, is more general in the sense of being 
able to provide an indication of how selection operates on endosperm alleles at any frequency, rather than just for $p \rightarrow 0$.

The assumptions are the same as for the previous model except for the following. Let " $E$ " be the more "selfish" of two endosperm alleles: $E E E$ endosperms take more PI than eee endosperms, giving their embryos an added fitness benefit of $b$ and imposing a total fitness cost of $c$ on half-sibling embryos. Eee endosperms give benefit $h_{1} b$ to their own embryos and cost $h_{1} c$ to half-sib embryos, and the comparable values for $E E e$ endosperms are $h_{2} b$ and $h_{2} c$. This differs slightly from the previous model in which subsequent units of PI taken yield diminishing increases in benefit, but linearly increasing costs. I assume further that the effeets of costs and benefits on fitness are additive (i.e., add benefits, subtract costs) and that the costs of "selfish" aquisition of PI are distributed randomly among half-siblings with respect to their genotypes.

Table 2 lists the endosperm/embryo genotypes (with maternal alleles in heterozygotes), their frequencies, and the expected benefits and costs the

\section{TABLE 2}

Expected costs and benefits to embryos due to their own endosperms and to the endosperms of other seeds on the same plant, assuming random mating so that embryos are half sibs to each other. The frequency of the more "selfish" endosperm allele, E, is p. The components of the expected cost are classified according to whether or not other endosperms share the maternal allele identical by descent (i.b.d.) with the embryo in question (maternal alleles are in heterozygous genotypes). The expected cost due to an endosperm with no alleles i.b.d. is $X=$ $\mathrm{p}^{2} \mathrm{c}+\mathrm{p}(1-\mathrm{p})\left(\mathrm{h}_{1}+\mathrm{h}_{2}\right) \mathrm{c}$. See text for discussion.

\begin{tabular}{cccccc}
\hline & & $\begin{array}{c}\text { gain } \\
\text { from own } \\
\text { endosperm }\end{array}$ & \multicolumn{3}{c}{$\begin{array}{c}\text { loss due to endosperms with: } \\
\text { maternal i.b.d. }\end{array}$} \\
genotype & freq. & paternal E & paternal $e$ & $\begin{array}{c}\text { maternal } \\
\text { not } \\
\text { i.b.d. }\end{array}$ \\
\hline$E E E / E E$ & $p^{2}$ & $b$ & $\frac{1}{2} p c$ & $\frac{1}{2}(1-p) h_{2} c$ & $\frac{1}{2} X$ \\
$E E \mathrm{e} / E \mathrm{e}$ & $p(1-p)$ & $h_{2} b$ & $\frac{1}{2} p c$ & $\frac{1}{2}(1-p) h_{2} c$ & $\frac{1}{2} X$ \\
Eee $/ E e$ & $p(1-p)$ & $h_{1} b$ & $\frac{1}{2} p h_{1} c$ & 0 & $\frac{1}{2} X$ \\
$e e e / e e$ & $(1-p)^{2}$ & 0 & $\frac{1}{2} p h_{1} c$ & 0 & $\frac{1}{2} X$ \\
\hline
\end{tabular}

embryo receives from its own endosperm and from the other endosperms on the same maternal plant. The costs are calculated from the probabilities of occurrence of the various endosperm genotypes conditioned on the one known maternal allele. For example, an $E$ e offspring has a mother with an $E$ allele and an unknown (randomly chosen) allele. Therefore, half of its sibling endosperms will have two identical-by-descent $E$ alleles, and a fraction $p$ (the population frequency of $E$ ) of these will also receive an $E$ through pollen, making an $E E E$ endosperm. If there are $n$ offspring per maternal parent, the average $E$ e offspring will have $\frac{1}{2} p(n-1)$ sibling endosperms formed in the above manner, each imposing a cost, $c$, on its $n-1$ siblings. The expected cost from these endosperms to a single $E$ e is therefore $\frac{1}{2} p c$. Other components of the expected cost are calculated in similar fashion. Costs and benefits may not accrue to the same individuals if, as in the previous model, the cost takes the form of total loss of fitness for some future siblings. But the values in table 2 still represent the average changes in fitness. 
I define the net gain, $G$, for each offspring genotype as its expected benefit minus its expected costs. $G$ differs from the usual selective coefficient in being constructed as an addition to fitness rather than a subtraction from it, and because it is convenient not to make the standard designation of $s=0$ for the fittest genotype.

With weak selection ( $b$ and $c$ small), $E$ will increase in frequency if the average gain experienced by $E$ alleles exceeds the average gain for all alleles or

$$
p \mathrm{G}_{\mathrm{EE}}+\frac{1-p}{2} \cdot \mathrm{G}_{\mathrm{Ee}}+\frac{1-\mathrm{p}}{2} \cdot \mathrm{G}_{\mathrm{Ee}}>\overline{\mathrm{G}}
$$

where $\overline{\mathrm{G}}$ is the average gain, $p b+p(1-p)\left(h_{1}+h_{2}\right)+\mathrm{X}$, and the coefficients of the L.H.S. are the fractions of the $E$ alleles that are found in each offspring genotype. Substitution of the G values (and X) into (10), and some algebra, shows that $\mathrm{E}$ is favoured when

$$
b\left[2\left(h_{1}+h_{2}\right)+4 p\left(1-h_{1}-h_{2}\right)\right]>c\left[h_{2}+p\left(1-h_{1}-h_{2}\right)\right] .
$$

When the $E$ allele is rare $(p \rightarrow 0)$, this gives the same result as the first model (equation (5)).

Some values of $h_{1}$ and $h_{2}$ for which condition (11) is frequency-independent are listed in table 3 . In general, however, the selective condition is

TABLE 3

Frequency-independent conditions, from formula 11 , for selection to favour a "selfish" endosperm allele that, when homozygous, gives its own embryo a benefit (b) and half-sibling embryos a cost (c). $h_{1}$ and $h_{2}$ denote the fractional expression of endosperms with one and two of the alleles. The last column relates the selective condition to those predicted on the basis of relatedness (Table 1)

\begin{tabular}{ccccc}
\hline$h_{1}$ & $h_{2}$ & label & selected when & behaves like \\
\hline 0 & 0 & recessive & $b>c / 4$ & offspring \\
1 & 1 & dominant & $b>c / 4$ & offspring \\
0 & 1 & threshold & $b>c / 2$ & gametophyte \\
$\frac{1}{3}$ & $\frac{2}{3}$ & additive & $b>c / 3$ & endosperm \\
\hline
\end{tabular}

frequency-dependent and two interesting possibilities arise: (1) that some alleles can invade but cannot be fixed (polymorphism), and (2) that some alleles that are not favoured at low frequencies may be favoured if they drift to a high enough frequency. These possibilities can be explored by determining how the threshold value of $b / c$ changes with $p$. From (11), $E$ is selected if

$$
\frac{b}{c}>\frac{h_{2}+p\left(1-h_{1}-h_{2}\right)}{2\left(h_{1}+h_{2}\right)+4 p\left(1-h_{1}-h_{2}\right)}
$$

except that when the denominator of the R.H.S. $<0$, the sign of the inequality is reversed. The change in the threshold value of $b / c$ that can be selected, $k$, with respect to change in gene frequency is

$$
\frac{d k}{d p}=\frac{\left(1-h_{1}-h_{2}\right)\left(h_{1}-h_{2}\right)}{2\left(h_{1}+h_{2}+2 p-2 p h_{1}-2 p h_{2}\right)^{2}} .
$$


TABLE 4

Conditions under which an endosperm allele for taking more parental investment at the expense of half-sib embryos becomes harder or easier to select as its frequency increases. See text for discussion

\begin{tabular}{ccc}
\hline & \multicolumn{1}{c}{$h_{1}>h_{2}$} & $h_{1}>h_{2}$ \\
\hline $\begin{array}{c}\text { partly recessive } \\
\left(\bar{h}>\frac{1}{2}\right)\end{array}$ & $\frac{d k}{d p}<0$, easier & $\frac{d k}{d p}>0$, harder \\
$\begin{array}{c}\text { partly dominant } \\
\left(\frac{1}{2}<\bar{h}<1\right)\end{array}$ & $\frac{d k}{d p}>0$, harder & $\frac{d k}{d p}<0$, easier \\
$\begin{array}{c}\text { overdominant } \\
(1<\bar{h})\end{array}$ & $\frac{d k}{d p}>0$ & $\frac{d k}{d p}<0$ \\
& $0<p<\hat{p}$, harder & $0<p<\hat{p}$, easier \\
& $\hat{p}<p<1$, easier & $\hat{p}<p<1$, harder \\
\hline
\end{tabular}

Some of the information provided by (12) and (13) is summarised in table 4. Whether "selfish" $E$ alleles become easier or harder to select as their frequency rises depends on the signs of the two terms in the numerator of (13) and on the direction of inequality (12), determined by the sign of the denominator on the R.H.S. The selective behaviour is best classified into categories based on the average expression of heterozygous endosperms, $\bar{h}=\frac{1}{2}\left(h_{1}+h_{2}\right)$. As long as (12) holds, as it always does for what I term partly recessive $\left(\bar{h}<\frac{1}{2}\right)$ and partly dominant $\left(\frac{1}{2}<\bar{h}<1\right)$ alleles, then a threshold $b / c$ value that increases with frequency $(d k / d p>0)$ means that the selective condition (12) becomes harder to meet so that some alleles that can successfully invade will not be able to fix. If the threshold $b / c$ decreases with frequency $(d k / d p<0)$, the selective condition becomes easier to meet so that all invading alleles can fix and some alleles with $b / c$ values too small to permit invasion can be selected if they drift to a high enough frequency.

The same is true for overdominant $(1 \leqslant \bar{h})$ alleles as long as (12) holds. But for these alleles there exists a threshold frequency, $\hat{p}=$ $\left(h_{1}+h_{2}\right) / 2\left(h_{1}+h_{2}-1\right)$, above which the denominator of the R.H.S. becomes negative and the sign of the inequality reverses. Since an allele must now have a $b / c$ value below the threshold $k$, the meaning of the sign of $d k / d p$ reverses above $\hat{p}$ : when $d k / d p<0$ the selective condition becomes more stringent, and when $d k / d p>0$ it becomes less stringent.

Table 4, together with formula (11), gives a more complete basis for predicting endosperm behaviour than the stability conditions of the first model. Since selection is dependent on dosage, a complete understanding of endosperm behaviour will require knowledge of the values of the dosage parameters for endosperm genes, specifically for endosperm genes that affect the amount of PI taken. Little is known of such genes since seeds have not been studied with parent-offspring conflict in mind, but some tentative $a$ priori assumptions can be made.

The additional effect on an endosperm of possessing a single "selfish" allele, a second such allele, and a third copy are proportional to $h_{1}, h_{2}-h_{1}$, and $1-h_{2}$, respectively. It seems most plausible that the effect of possessing a second copy would usually be intermediate between the effects of the first and third copies. Clearly this rules out the $h_{1}>h_{2}$ cases, at least for nonoverdominant alleles, because $h_{2}-h_{1}$ would be negative and therefore not intermediate between $h_{1}$ and $1-h_{2}$, which are both positive. 
Assuming now that $h_{1}<h_{2}$, similar arguments can further narrow the range of plausible dosage parameters. For partly recessive alleles it is most likely that $h_{2}$ is more than twice as large as $h_{1}$, because otherwise the effect of the second copy $\left(h_{2}-h_{1}\right)$ would be less than the effects of the first $\left(h_{1}\right)$ and the third $\left(1-h_{2}\right)$. For this plausible class of partly recessive alleles, formula (12) shows that the threshold $k=b / c$ necessary for invasion lies between $\frac{1}{3}$ and $\frac{1}{2}$, that is, between the selective threshold predicted by relatedness and the less "selfish" gametophytic threshold (see table 1). However, for partly recessive alleles, the selective condition becomes less stringent as $p$ increases (table 4) so that drift may assist in the spread of some alleles that are too "selfish" to invade. The effect of partly recessive alleles on the actual behaviour of the endosperm is therefore probably closer to that predicted by relatedness than would be expected from the invasion thresholds alone.

The discontinuity in behaviour between completely recessive alleles (invasion threshold, $k=1 / 4)$ and partial recessives $\left(\frac{1}{3}<k<\frac{1}{2}\right)$ is not real. It arises because I have only considered partial recessives for which $h_{2}>2 h_{1}$. If we are to consider a continuous transition from alleles of this type to complete recessives $\left(h_{2}=h_{1}=0\right)$, we must cross a zone of partial recessives for which $h_{2}<2 h_{1}$, and the behaviour of these alleles would fill the apparent gap.

Partly dominant alleles behave in the opposite way. The most plausible class of these would have $h_{2}$ less than twice as large as $h_{1}$. Otherwise the effect of the second allele would be greater than the effects of both the first and third copies. When $2 h_{1}<h_{2}$, the invasion threshold from formula 12 lies between $\frac{1}{4}$ and $\frac{1}{3}$, that is, between the offspring threshold and the endosperm threshold predicted from relatedness. However, in this case, the selective condition becomes harder to meet as $p$ increases, so the closer an allele is to the more selfish end of the range of invasion criteria, the less it spreads. Only alleles with $b / c \geqslant \frac{1}{3}$ spread to fixation. Again the invasion criterion overestimates the extent to which the behaviour of the endosperm diverges from what is predicted from relatedness alone.

Overdominant alleles, when $h_{1}<h_{2}$, behave in a way similar to partly dominant alleles except that none of them can reach frequency $\hat{p}$ (the L.H.S. of formula 11 approaches zero as $p \rightarrow \hat{p}$ ). Perhaps the possibility of $h_{1}$ being greater than $h_{2}$ should not be discounted for overdominant alleles (though it is still less plausible than the alternative since three "selfish" alleles have greater effect than none). In this event, endosperm alleles can be selected that take even more PI than offspring alleles. Traditional explanations of the function of double fertilisation often posit an increased efficiency of the nutrient gathering tissue due to heterosis (e.g., Brink and Cooper, 1947). Whatever the merits of this view (see Queller, 1983 for discussion), it is now apparent that it would have to be modified to take account of the effects of heterosis on what kinds of alleles can be selected as well as its effects on physiological vigour.

\section{Discussion}

The models developed in this paper agree rather well with the predictions based on relatedness alone. Similar models by Law and Cannings (unpublished ms.) seem to show a less precise fit, although it is less easy to tell because they solve only for invasion criteria and because their models are 
formulated in terms of genotypic fitnesses rather than costs and benefits. One difference from the models developed here is that dominant alleles in embryos and endosperms invade more easily than recessive alleles. This difference, as well as others, is due to a difference in assumptions about the way PI is divided.

I have assumed that the cost of taking extra PI by some seeds is manifested only by the mother being able to rear fewer seeds in the future. In the first model this assumption is represented explicitly, e.g., the numbers of seeds differ on the two sides of formula 1 . In the second model, the assumption is represented indirectly by its consequence: the seeds bearing the cost will be a genetically random subset of the mother's progeny, those seeds she would have reared if she still had PI left. Since a random subset of seeds is affected, average relatedness should be relevant and Hamilton's rule works. Law and Cannings assume that each parent raises the same number of seeds, but that "selfish" seeds all get more PI than "unselfish" seeds. Under this assumption, sibling seeds are not affected at random, so Hamilton's rule fails. For example, a dominant "selfish" allele will always be expressed, so all endosperms bearing this allele will gain PI entirely at the expense of those which do not. This assumption is most plausible when a set of contemporary seeds compete for a fixed amount of PI, without affecting the amount of PI left over for later seeds (see Macnair and Parker, 1979, and Metcalf et al., 1979).

Which assumption is appropriate will depend on the biology of the particular species. For many species, excess PI taken by some seeds may affect both contemporary and later seeds so that a hybrid between the two models may be most appropriate. However, one cautionary note seems necessary for the intrabrood model. Macnair and Parker (1979) have shown that, even when offspring affect only their contemporary siblings, relatedness arguments are correct for alleles of low penetrance. The more rarely an allele is expressed, the more random is the subset of siblings that feels the costs. The same should be true for genes in gametophytes and endosperms. Thus, when penetrance is low, the models in this paper are probably more appropriate even when only contemporary seeds are affected.

The effect of dosage on endosperm selection, which can lead to frequency dependence of the selective condition (11), represents the only departure from Hamilton's rule (or actually from the modified rule, $b r_{b}>c r_{c}$ for helping one relative at the expense of another) shown by the models presented in this paper. While Hamilton's rule does not predict these phenomena, dependence of the selective condition on dominance and frequency has turned up in many genetic models of sibling interactions (Michod, 1982). This is usually due to benefits or costs whose magnitude depends in some way on the frequency of altruists in the sibship (e.g., Charlesworth, 1978). Strong selection, and the concomitant distortion of Hardy-Weinberg frequencies, can also cause deviations from Hamilton's rule (e.g., Uyenoyama and Feldman, 1981). However, the models in this paper assumed constant, additive costs and benefits as well as weak selection and, as expected under these circumstances, Hamilton's rule is correct for offspring selection. The deviations for endosperm selection must therefore be due to a different cause.

The peculiarities of endosperm selection do have some affinity with those arising from another cause of deviations from Hamilton's rule: inbreeding. Hamilton's rule (using the regression coefficient of relatedness- 
Hamilton, 1972) does work for inbreeding with additive dosage effects $\left(h=\frac{1}{2}\right)$, but is not precise for non-additive dosage (Michod, 1982). Under inbreeding, an individual's two alleles are not inherited independently. As a consequence, genes in heterozygotes and homozygotes do not have the same probabilities of having copies identical by descent in siblings. Therefore, in general, the selective threshold of $b / c$ changes as the relative frequencies of heterozygotes and homozygotes change with $p$, and selection differs for dominant and recessive alleles because the latter are expressed only in homozygotes. However, if dosage is additive, the effect of two alleles in homozygous condition is exactly twice the effect of one allele in heterozygous condition. All copies of the allele therefore have the same effect and the use of the average relatedness coefficient gives the correct result.

This kind of effect also appears in selection of endosperm alleles (even with outbreeding). This is because the endosperm is always inbred in the sense that its two maternal alleles are not inherited independently. Consider the frequency-independent conditions in table 3 . For the additive case $\left(h_{1}=\frac{1}{3}, h_{2}=\frac{2}{3}\right)$ each copy of the allele adds the same cost and benefit, regardless of genotype, so the average relatedness produces a correct result $(b>c / 3)$. Of the non-additive cases, the "threshold" type of allele $\left(h_{1}=0\right.$, $h_{2}=1$ ) is the most transparent. Here, a "selfish" endosperm allele is expressed only when two or more copies are present, i.e., if and only if the gametophyte has the "selfish" allele. Selection therefore operates exactly as if such alleles were expressed in the gametophyte. Put another way, relatedness through pollen (or rather, the fact that there is no relatedness through pollen) is completely irrelevant because pollen-derived alleles never change the behaviour of the endosperm.

The dominant and recessive cases may be understood in similar ways. A recessive endosperm allele is expressed in exactly the same circumstances as a recessive embryo allele (i.e., the endosperm has 3 copies if and only if the embryo has 2). It is therefore selected exactly as if it were an embryo recessive. An endosperm dominant is expressed under exactly the same conditions in which an embryo dominant would be expressed, and is therefore selected as if it were an embryo dominant. The critical point is that the second copy of the maternally-derived endosperm allele is not independent of the first. It becomes important in selection only when it adds something to the effect of the first.

From (12) an endosperm allele can invade when $b / c>h_{2} / 2\left(h_{1}+h_{2}\right)$. This condition is harder to meet than the one predicted by average relatednesses $\left(b / c>\frac{1}{3}\right)$ when $h_{1}<\frac{1}{2} h_{2}$. This is because maternally-derived alleles (in $E E \mathrm{e}$ ) have a greater average effect than paternally-derived alleles (in $E$ ee), so that relatedness through the maternal side is more important. When $h_{1}>\frac{1}{2} h_{2}$, paternally-derived alleles have the greater average effect and the consequent greater importance of relatedness through the paternal side (or lack of it) makes it harder for such alleles to invade than would be expected on the basis of average relatedness. Note that I am not claiming that endosperm alleles can generally tell whether they are maternally or paternally derived and act accordingly. Selection simply acts on the effects of alleles, but dosage can cause alleles from one parent to have more effect than alleles from the other.

If the deviations from Hamilton's rule in endosperm selection are analogous to those arising under inbreeding, it should be possible to correct for them in the same way. Hamilton's rule is valid even for the non-additive 
inbreeding case (Michod, 1982) provided we use the general relatedness coefficient, $\operatorname{Cov}\left(\mathrm{Y}_{g}, \mathrm{X}_{p}\right) / \operatorname{Cov}\left(\mathrm{X}_{\mathrm{g}}, \mathrm{X}_{p}\right)$, first derived by Michod and Hamilton (1980). Here $X_{p}$ is the phenotypic value of the altruist, while $X_{g}$ and $\mathrm{Y}_{g}$ are the genotypic values of the altruist and beneficiary. Since the endosperm helps one relative at the expense of another, the relevant version of Hamilton's rule is $b>c r_{c} / r_{b}$, and the covariance form of the relatedness ratio is:

$$
\frac{\operatorname{cov}\left(C_{g}, X_{p}\right) / \operatorname{cov}\left(X_{g}, X_{p}\right)}{\operatorname{cov}\left(B_{g}, X_{p}\right) / \operatorname{cov}\left(X_{g}, X_{p}\right)}=\frac{\operatorname{cov}\left(C_{g}, X_{p}\right)}{\operatorname{cov}\left(B_{g}, X_{p}\right)}
$$

Here $\mathrm{X}_{p}$ is the phenotypic value of the endosperm which assumes values $0, h_{1}, h_{2}$, and 1 for endosperms with $0,1,2$, and 3 copies of the "selfish" allele. $C_{g}$ and $B_{g}$ are the genotypic values of the embryos receiving the cost and benefit, and assume the values $0, \frac{1}{2}$, and 1 for embryos with 0,1 , and 2 copies of the allele. When the allele is at frequency $p$ and causes endosperms to take more PI for their own embryos at the expense of randomly chosen half-sibling embryos,

$$
\begin{aligned}
\operatorname{cov}\left(\mathrm{C}_{g}, \mathrm{X}_{p}\right)= & \frac{1}{2}\left[p^{2}\left(1-\overline{\mathrm{X}}_{p}\right)\left(1-\overline{\mathrm{C}}_{g}\right)+p(1-p)\left(h_{2}-\overline{\mathrm{X}}_{p}\right)\left(\frac{1}{2}-\overline{\mathrm{C}}_{g}\right)\right. \\
& \left.+p(1-p)\left(h_{1}-\overline{\mathrm{X}}_{p}\right)\left(-\overline{\mathrm{C}}_{g}\right)+(1-p)^{2}\left(-\overline{\mathrm{X}}_{p}\right)\left(-\overline{\mathrm{C}}_{g}\right)\right], \\
\operatorname{cov}\left(\mathrm{B}_{g}, \mathrm{X}_{p}\right)= & p^{2}\left(1-\overline{\mathrm{X}}_{p}\right)\left(1-\overline{\mathrm{B}}_{\mathrm{g}}\right)+p(1-p)\left(h_{2}-\overline{\mathrm{X}}_{p}\right)\left(\frac{1}{2}-\overline{\mathrm{B}}_{g}\right) \\
& +p(1-p)\left(h_{1}-\overline{\mathrm{X}}_{p}\right)\left(\frac{1}{2}-\overline{\mathrm{B}}_{g}\right)+(1-p)^{2}\left(-\overline{\mathrm{X}}_{p}\right)\left(-\overline{\mathrm{B}}_{g}\right),
\end{aligned}
$$

where the means are $\overline{\mathrm{X}}=p\left(h_{1}+h_{2}\right)+p^{2}\left(1-h_{1}-h_{2}\right)$ and $\overline{\mathrm{C}}_{\mathrm{g}}=\overline{\mathrm{B}}_{\mathrm{g}}=p$. Solving for $r_{c} / r_{b}$ yields

$$
\frac{h_{2}+p\left(1-h_{1}-h_{2}\right)}{2\left(h_{1}+h_{2}\right)+4 p\left(1-h_{1}-h_{2}\right)} .
$$

Since this is the same as the coefficient of the R.H.S. of formula 12, the variant of Hamilton's rule, $b>c r_{c} / r_{b}$ holds for all frequencies and all dosage values when relatednesses are expressed in the covariance form. This is consistent with the peculiarities of endosperm selection stemming from what is essentially an inbreeding effect.

In conclusion, the models in this paper verify the prediction, based on relatedness arguments, that the endosperm and the gametophyte are in some sense independent players in the evolutionary game and not simply extensions of either the maternal plant or of the embryo. They also support the prediction that the four seed components can be ranked, in order of decreasing degree of selection for taking more parental investment, as embryo, endosperm, gametophyte, and maternal plant. The quantitative fit to simple relatedness models is also fairly close. Explanations of features of seed development in terms of relatedness (Westoby and Rice, 1982; Queller, 1983) are therefore soundly based, at least for the conditions in which the present models are applicable. Two qualifications should, however, be kept in mind. First, undue emphasis should not be placed on relatedness to the exclusion of the cost and benefit terms; the different seed components probably have different opportunities to aid their embryo. Second, each model presented here involves only one gene expressed in only one of the seed components. While such models can predict how much 
parental investment each seed component "favours" for its embryo, they do not predict how the conflict among the seed components is resolved.

Acknowledgements. This research was made possible by a NATO postdoctoral fellowship. I thank Brian Charlesworth for criticism of the manuscript and for the idea that dosage should be important in endosperm selection. Richard Law generously provided an unpublished manuscript and useful discussion.

\section{REFERENCES}

Brink, R. A. AND COOPER, D. C. 1947. The endosperm in seed development. Bot. Rev., 13, 423-541.

CHARLESWORTH, B. 1978. Some models of the evolution of altruistic behaviour between siblings. J. Theoret. Biol., 72, 297-319.

CHARNOV, E. L. 1979. Simultaneous hermaphroditism and sexual selection. Proc. Nat. Acad. Sci. USA, 76, 2480-2484.

HAMILTON, w. D. 1964. The genetical evolution of social behaviour. J. Theoret. Biol., 7, 1-52.

HAMILTON, w. D. 1972. Altruism and related phenomena, mainly in the social insects. Ann. Rev. Ecol. Syst., 3, 192-232.

LEVITT, P. R. 1975. General kin selection models for genetic evolution of sib altruism in diploid and haplodiploid species. Proc. Nat. Acad. Sci. USA, 72, 4531-4535.

MACNAIR, M. R. AND PARKER, G. A. 1978. Models of parent-offspring conflict. II. Promiscuity. Anim. Behav., 26, 111-122.

MACNAIR, M. R. AND PARKER, G. A. 1979. Models of parent-offspring conflict. III. Intrabrood conflict. Anim. Behav., 27, 1202-1209.

MAHESHWARI, P. 1950, An Introduction to the Embryology of the Angiosperms. McGraw-Hill, New York.

MAYNARD SMITH, J. 1965. The evolution of alarm calls. Amer. Nat., 94, 59-63.

MAYNARD SMITH, J. 1982. Evolution and the Theory of Games. Cambridge Univ. Press, Cambridge.

METCALF, R. A., STAMPS, J. A. AND KRISHNAN, v. v. 1979. Parent-offspring conflict which is not limited by degree of kinship. J. Theoret. Biol. 81, 99-107.

MICHOD, R. E. 1982. The theory of kin selection. Ann. Rev. Ecol. Syst., 13, 23-55.

MICHOD, R. E. AND HAMILTON, W. D. 1980. Coefficients of relatedness in sociobiology. Nature, 288, 694-697.

PARKER, G. A. AND MACNAIR, M. R. 1978. Models of parent-offspring conflict. I. Monogamy. Amin. Behav., 26, 97-111.

QUELLER, D. C. 1983. Kin selection and conflict in seed maturation. J. Theoret. Biol., 100, 153-172.

SMITH, C. C. AND FRETWELL, S. D. 1974. The optimal balance between size and number of offspring. Amer. Nat., 108, 499-506.

Trivers, R. L. 1974. Parent-offspring conflict. Amer. Zool., 14, 249-264.

UYENOYAMA, M. K. AND FELDMAN, M. W. 1981. On relatedness and adaptive topography in kin selection. Theoret. Pop. Biol., 19, 87-123.

WEST-EBERHARD, M. J. 1975. The evolution of social behaviour by kin selection. $Q$. Rev. Biol., 50, 1-33.

WESTOBY, M. AND RICE, B. 1982. Evolution of seed plants and inclusive fitness of plant tissues. Evolution, 36, 713-724.

WILliaMS, G. C. AND WILliAMS, D. C. 1957. Natural selection of individually harmful social adaptations among sibs with special reference to social insects. Evolution, 11, 32-39. 